\title{
Association of systolic and diastolic blood pressure and all cause mortality in people with newly diagnosed type 2 diabetes: retrospective cohort study
}

\author{
(C) $\mathbb{Q} \Theta$ OPEN ACCESS
}

\author{
Eszter Panna Vamos academic clinical fellow ${ }^{1}$, Matthew Harris academic clinical lecturer ${ }^{1}$, \\ Christopher Millett clinical senior lecturer ${ }^{1}$, Utz J Pape statistician ${ }^{1}$, Kamlesh Khunti professor ${ }^{2}$, \\ Vasa Curcin research fellow ${ }^{3}$, Mariam Molokhia reader ${ }^{4}$, Azeem Majeed professor ${ }^{1}$
}

${ }^{1}$ Department of Primary Care and Public Health, Imperial College London, London W6 8RP, UK; ${ }^{2}$ Department of Health Sciences, University of Leicester, Leicester, UK; ${ }^{3}$ Department of Computing, Imperial College London; ${ }^{4}$ Department of Primary Care and Public Health Sciences, King's College London, London, UK

\begin{abstract}
Objective To examine the effect of systolic and diastolic blood pressure achieved in the first year of treatment on all cause mortality in patients newly diagnosed with type 2 diabetes, with and without established cardiovascular disease.

Design Retrospective cohort study.

Setting United Kingdom General Practice Research Database, between 1990 and 2005.

Participants 126092 adult patients (age $\geq 18$ years) with a new diagnosis of type 2 diabetes who had been registered with participating practices for at least 12 months.

Main outcome measure All cause mortality.

Results Before diagnosis, 12379 (9.8\%) patients had established cardiovascular disease (myocardial infarction or stroke). During a median follow-up of 3.5 years, we recorded 25495 (20.2\%) deaths. In people with cardiovascular disease, tight control of systolic $(<130 \mathrm{~mm} \mathrm{Hg})$ and diastolic $(<80 \mathrm{~mm} \mathrm{Hg}$ ) blood pressure was not associated with improved survival, after adjustment for baseline characteristics (age at diagnosis, sex, practice level clustering, deprivation score, body mass index, smoking, $\mathrm{HbA}_{1 \mathrm{c}}$ and cholesterol levels, and blood pressure). Low blood pressure was also associated with an increased risk of all cause mortality. Compared with patients who received usual control of systolic blood pressure $(130-139 \mathrm{~mm} \mathrm{Hg})$, the hazard ratio of all cause mortality was 2.79 (95\% confidence interval 1.74 to $4.48, \mathrm{P}<0.001)$ for systolic blood pressure at $110 \mathrm{~mm} \mathrm{Hg}$. Compared with patients who received usual control of diastolic blood pressure $(80-84 \mathrm{~mm} \mathrm{Hg})$, the hazard ratios were 1.32 ( 1.02 to $1.78, \mathrm{P}=0.04)$ and 1.89 ( 1.40 to $2.56, \mathrm{P}<0.001)$ for
\end{abstract}

diastolic blood pressures at $70-74 \mathrm{~mm} \mathrm{Hg}$ and lower than $70 \mathrm{~mm} \mathrm{Hg}$, respectively. Similar associations were found in people without cardiovascular disease. Subgroup analyses of people diagnosed with hypertension and who received treatment for hypertension confirmed initial findings.

Conclusion Blood pressure below 130/80 mm Hg was not associated with reduced risk of all cause mortality in patients with newly diagnosed diabetes, with or without known cardiovascular disease. Low blood pressure, particularly below $110 / 75 \mathrm{~mm} \mathrm{Hg}$, was associated with an increased risk for poor outcomes.

\section{Introduction}

Clinical guidelines recommend maintaining blood pressure levels to below 140/90 $\mathrm{mm} \mathrm{Hg}$ in people with hypertension in the general population. ${ }^{12}$ Guidelines recommend further lowering the blood pressure to a treatment goal of below 130/80 $\mathrm{mm} \mathrm{Hg}$ in high risk patients, including people with diabetes, cerebrovascular and coronary arterial disease, or kidney disease. ${ }^{3}$ The initiation of antihypertensive therapy is indicated in high risks groups, even if their blood pressure is in the normal range, for further cardiovascular benefit. ${ }^{12}$

This lower treatment target in patients at high risk of cardiovascular disease was largely based on the results of early randomised trials such as the United Kingdom Prospective Diabetes Study (UKPDS) and Hypertension Optimal Treatment (HOT) trial. These trials showed major reductions in cardiovascular outcomes in the groups receiving tight control of blood pressure compared with those receiving conventional 
control..$^{4-7}$ Furthermore, evidence from epidemiological studies suggests that cardiovascular risk starts to increase above a blood pressure level of $115 / 75 \mathrm{~mm} \mathrm{Hg} .{ }^{8}$ This evidence has led to the consensus that there is no lower threshold for blood pressure lowering, supporting the idea that "the lower the better".

Aggressive targets for blood pressure treatment in type 2 diabetes guidelines have recently been questioned, ${ }^{10}{ }^{11}$ because the recommended targets are well below those found in the trials on which the guidelines are based ${ }^{4}{ }^{12}$ The Action to Control Cardiovascular Risk in Diabetes (ACCORD) trial did not show further cardiovascular benefits of antihypertensive therapy through reducing systolic blood pressure below $130 \mathrm{~mm} \mathrm{Hg}$ in people with diabetes. ${ }^{213}$ To date, there is little evidence indicating the cardiovascular protective effect of blood pressure targets lower than 130/80 mm Hg in patients with diabetes. ${ }^{10}$ Importantly, some studies suggest that blood pressure lowering that is too aggressive in high risk patients could do harm rather than provide cardiovascular protection. ${ }^{14}$

There are limited data available from population based studies on the association between blood pressure and mortality in people with newly diagnosed type 2 diabetes. Furthermore, little is known about these associations in people with diabetes and cardiovascular disease. This retrospective study aimed to determine the association between systolic and diastolic blood pressure in the first year of treatment and the risk of all cause mortality, in a large primary care based cohort of patients with newly diagnosed type 2 diabetes, with and without established cardiovascular disease.

\section{Methods}

The General Practice Research Database holds anonymised, longitudinal primary care records of approximately $5 \%$ of the population registered with a general practice in the UK. ${ }^{16}$ Patients registered with general practices participating in the database represent a population that is similar to that of the UK. ${ }^{16}$ Participating practices follow an agreed protocol for the collection of demographic, clinical, laboratory, and prescription data, and regularly submit anonymised records to the database.

\section{Study population}

From the General Practice Research Database, we extracted the medical records of all adult patients (age $\geq 18$ years) with a new diagnosis of type 2 diabetes between 1 January 1990 and 31 December 2005, and who had been registered with participating practices for at least 12 months. Patients were identified using both diagnostic (C10) and management (66A) Read and Oxford Medical Information System codes for diabetes. ${ }^{17}$ We excluded patients diagnosed under the age of 35 years who were prescribed insulin within three months of diagnosis and who were not prescribed oral hypoglycaemic agents for longer than three months, because these patients were likely to have type 1 diabetes. We also excluded patients with a diagnosis of heart failure and an echocardiogram supporting the diagnosis to avoid reverse causality, because these patients tend to have lower blood pressure levels than those without heart failure.

\section{Study variables}

The primary outcome of interest was all cause mortality as identified by codes for death or for transfer out of practice due to death in the General Practice Research Database. Patients were followed from the date of diagnosis until death or the end of the study (31 December 2005). Patients who did not die during the follow-up period and those who left the practice were censored at the last visit. Covariates in our study included age at diagnosis, sex, socioeconomic status, smoking status (classified as never, current or ex-smoker), body mass index, blood pressure readings, and laboratory variables (total cholesterol, $\mathrm{HbA}_{1 \mathrm{c}}$ ). Socioeconomic status was assigned to each patient using the Index of Multiple Deprivation 2004, the most commonly used method of measuring socioeconomic status of a neighbourhood in the UK, based on the postal codes of general practices involved. ${ }^{18} \mathrm{We}$ identified treatments of individual patients from prescription records: antihypertensive drugs, oral hypoglycaemic agents, insulin, and lipid lowering and antiplatelet drugs. Patients were categorised into two groups, based on whether or not they had a cardiovascular event (defined as stroke or myocardial infarction) recorded before the diagnosis of diabetes.

\section{Blood pressure profiles}

All patients had their blood pressure measured at least once during the first year after diagnosis of diabetes. Patients were categorised into three groups by readings of mean systolic and diastolic blood pressure using all except for their baseline measurement (baseline blood pressure measurements were defined as that measured in the diagnostic visit or within 3 months from that date-we excluded the baseline measurements due to regression to the mean). The three groups were: tight control (systolic blood pressure $<130 \mathrm{~mm} \mathrm{Hg}$; diastolic blood pressure $<80 \mathrm{~mm} \mathrm{Hg}$ ), usual control (130 to $<140 \mathrm{~mm} \mathrm{Hg} ; 80$ to $<85 \mathrm{~mm} \mathrm{Hg}$ ), and uncontrolled ( $\geq 140 \mathrm{~mm} \mathrm{Hg} ; \geq 85 \mathrm{~mm} \mathrm{Hg}$ ). To explore the risk associated with systolic and diastolic blood pressure, we further categorised the tight and uncontrolled groups in 10 and $5 \mathrm{~mm} \mathrm{Hg}$ segments, resulting in seven groups of systolic and diastolic blood pressure.

\section{Statistical analysis}

We compared baseline characteristics of study patients by cardiovascular disease status. Group differences were tested using the $\chi^{2}$ test for categorical variables, Student's $t$ test for normally distributed variables, or Mann-Whitney test for skewed continuous variables, as appropriate.

We assessed survival estimates with Kaplan-Meier plots. Equality of survival distributions for the different levels of systolic and diastolic blood pressure categories were tested using log rank (Mantel-Cox) tests. We used Cox proportional hazard regression models to estimate hazard ratios with $95 \%$ confidence intervals of all cause mortality for groups of systolic and diastolic blood pressure, using the usual control groups as references. Patients treated by the same general practice are expected to have more similar outcomes than patients treated in different practices. We used a robust estimator for the standard error to control for the clustering of patients within practices. Models were adjusted for sex, age at baseline, deprivation score, body mass index, smoking status, baseline levels of cholesterol and $\mathrm{HbA}_{1 \mathrm{c}}$, and blood pressure at baseline. To test the validity of the findings, we undertook two subgroup analyses restricting the models to patients who received treatment for hypertension, or had a diagnosis of hypertension at baseline.

Proportional hazards analyses assume that the ratio of mortality risk for a predictor variable remains constant (that is, proportional) over time. We tested this assumption graphically for each covariate by examining plots of $\log (-\log$ survival time) against log survival time, and of Schoenfeld residuals against survival time. Furthermore, we tested the assumption of proportionality by using generalised linear regression of the 
scaled Schoenfeld residuals on functions of time to test for a non-zero slope. This analysis revealed the violation of the proportionality assumption for levels of systolic and diastolic blood pressure in the unadjusted models. Therefore, we showed the odds ratios and confidence intervals obtained from conditional logistic regression models for the univariate association between blood pressure levels and mortality.

In the adjusted Cox proportional hazards models, the assumption was violated with regards to deprivation score, which was corrected by modelling deprivation score as a time-varying covariate. These changes did not qualitatively alter the estimates for variables of interest. When testing the assumption in the final model examining diastolic blood pressure among people with cardiovascular disease, $\mathrm{HbA}_{\mathrm{lc}}$ levels reached significance. However, the plot of Schoenfeld residuals versus time for this covariate did not seem to indicate a gross violation of the proportionality assumption.

In all cases, a two sided $\mathrm{P} \leq 0.05$ was considered statistically significant. We did statistical analyses using Stata version 11.0.

\section{Results}

\section{Baseline characteristics}

We included 126092 people, registered with 422 participating practices and who were diagnosed with type 2 diabetes between 1990 and 2005. Of these patients, 12379 (9.8\%) had known cardiovascular disease before diagnosis of diabetes. The median follow-up time was 3.5 years, during which 25495 deaths (20.2\%) were recorded, corresponding to an event rate of 48.3 per 1000 patient years in the study population.

The overall mortality was $28.6 \%(n=3535)$ in people with cardiovascular disease and $19.3 \%(n=21960)$ in those without cardiovascular disease (event rates 82.9 and 45.3 per 1000 patient years, respectively). Table $1 \Downarrow$ shows the baseline characteristics of patients with and without pre-existing cardiovascular disease. People with cardiovascular disease were significantly older; were more likely to be male and ex-smokers; and had lower levels of body mass index, $\mathrm{HbA}_{1 \mathrm{c}}$, and cholesterol at diagnosis than patients without cardiovascular disease. They were also more likely to have antihypertensive, lipid lowering, and antiplatelet treatment prescribed and less likely to receive antidiabetic drugs during the study period. Use of diuretics followed by angiotensin converting enzyme inhibitors (ACEI) or angiotensin II receptor blockers (ARB) were the most commonly prescribed antihypertensive drugs at baseline. Significantly higher prescription rates were recorded in patients with cardiovascular disease than in those without (diuretics, $5538(44.7 \%) v 31501$ (27.7\%); ACEI or ARB, $4630(37.4 \%)$ $v 25147$ (22.1\%), $\mathrm{P}<0.001$ for both).

\section{Blood pressure control according to cardiovascular disease status}

At the time of diagnosis of diabetes, patients with established cardiovascular disease had significantly lower mean levels of blood pressure than those without cardiovascular disease (table 1). In both people with and without cardiovascular disease, the mean values of systolic and diastolic blood pressure decreased significantly during the first year after diagnosis compared with blood pressure recordings at baseline (paired $t$ test, $\mathrm{P}<0.001$ for both).

The mean levels of systolic and diastolic blood pressure achieved during the first year after diagnosis (not including the baseline recordings) were significantly lower in people with cardiovascular disease than in those without. Accordingly, patients with cardiovascular disease were more likely to be recorded to have tight controls of blood pressure and reduced rates of uncontrolled blood pressure compared with patients without cardiovascular disease (table 1).

\section{Death from all causes according to blood pressure control}

Figure $1 \Downarrow$ shows hazard ratios for tight control (systolic blood pressure <130 mm Hg; diastolic blood pressure < $80 \mathrm{~mm} \mathrm{Hg}$ ), usual control (130-139 $\mathrm{mm} \mathrm{Hg} ; 80$ to $<85 \mathrm{~mm} \mathrm{Hg}$ ), and uncontrolled ( $\geq 140 \mathrm{~mm} \mathrm{Hg} ; \geq 85 \mathrm{~mm} \mathrm{Hg}$ ). In people with cardiovascular disease, tight control was associated with significantly reduced survival compared with the normal and uncontrolled groups, in unadjusted Kaplan-Meier analyses (log rank test, $\mathrm{P}<0.001$ for all; fig $2 \Downarrow$ ). In univariate models, because of the proportional hazards violation, we used logistic regression models to obtain odds ratios and confidence intervals. In these models, all cause mortality was related to systolic and diastolic blood pressure in a $U$ shaped pattern, with increased risk of death in the tight control and uncontrolled groups compared with the usual control group (table $2 \Downarrow$ ).

After adjustment for baseline characteristics in the Cox proportional hazards models, the increased risk of all cause mortality persisted for tight blood pressure control. In patients with cardiovascular disease, the hazard ratio was $2.79(95 \%$ confidence interval 1.74 to $4.48, \mathrm{P}<0.001)$ for systolic blood pressure lower than $110 \mathrm{~mm} \mathrm{Hg}, 1.32$ (1.02 to $1.78, \mathrm{P}=0.04$ ) for diastolic blood pressure $70-74 \mathrm{~mm} \mathrm{Hg}$, and 1.89 (1.40 to 2.56, $\mathrm{P}<0.001$ ) for diastolic blood pressure lower than $70 \mathrm{~mm}$ $\mathrm{Hg}$ (fig 1). Hazard ratios for other groups of tight control also indicated increased risk of mortality, but these associations did not reach statistical significance. Uncontrolled systolic blood pressure levels did not show significant association with the outcome.

In people without cardiovascular disease, we observed a similar pattern for systolic and diastolic blood pressure in unadjusted models (fig 2, table 2). After Cox model adjustment for baseline characteristics, we also saw an increased risk for death in tight control groups compared with usual control groups. The hazard ratio was 1.58 (95\% confidence interval 1.27 to $1.96, \mathrm{P}<0.001)$ for systolic blood pressure $110-119 \mathrm{~mm} \mathrm{Hg}, 2.42$ (1.6 to 3.52, $\mathrm{P}<0.001)$ for systolic blood pressure lower than $110 \mathrm{~mm} \mathrm{Hg}$, 1.17 (1.02 to $1.34, \mathrm{P}=0.02$ ) for diastolic blood pressure 70-74 $\mathrm{mm} \mathrm{Hg}$, and 1.54 (1.33 to $1.77, \mathrm{P}<0.001$ ) for diastolic blood pressure lower than $70 \mathrm{~mm} \mathrm{Hg}$ (fig 1). Patients with diastolic blood pressure $75-79 \mathrm{~mm} \mathrm{Hg}$ were associated with the lowest predicted risk for mortality (hazard ratio 0.87 (0.76 to 0.99 ), $\mathrm{P}=0.04$ ). Uncontrolled systolic and diastolic blood pressure levels were not significantly associated with the outcome compared with the usual control groups in multivariate Cox models (fig 1). In the total population, we found qualitatively similar results compared with people without cardiovascular disease (table 2, fig $3 \Downarrow$ ).

Subgroup analyses confirmed the findings of our initial observations. After restricting the analyses to patients who received medical treatment for hypertension and those who had a diagnosis of hypertension at diagnosis, we found qualitatively similar findings for mortality when comparing tight control with usual control, and comparing uncontrolled blood pressure with usual control in both people with and without cardiovascular disease (web appendices 1 and 2). 


\section{Discussion}

This observational study was undertaken to relate the levels of systolic and diastolic blood pressure achieved during the first year after diagnosis of diabetes to the risk of all cause mortality in a large cohort of patients with newly diagnosed type 2 diabetes. Our results show that in patients with diabetes and cardiovascular disease, systolic blood pressure below $110 \mathrm{~mm}$ $\mathrm{Hg}$ and diastolic blood pressure below $75 \mathrm{~mm} \mathrm{Hg}$ were associated with significantly increased risk of death. In patients with diabetes without established cardiovascular disease, systolic blood pressure below $120 \mathrm{~mm} \mathrm{Hg}$ and diastolic blood pressure below $75 \mathrm{~mm} \mathrm{Hg}$ were associated with a significant increased risk of mortality. These associations persisted when we restricted our analyses to patients who received treatment for hypertension and to those who had a diagnosis of hypertension at baseline.

\section{Comparison with other studies}

The risks of elevated blood pressure have been repeatedly demonstrated by clinical and epidemiological studies. ${ }^{81920}$ Many studies have described a gradual increase in the risk of mortality with increasing levels of systolic and diastolic blood pressure, with no lower thresholds for blood pressure. ${ }^{8}$ Despite the known benefits of blood pressure lowering and the effectiveness of medical treatment to reduce blood pressure, the optimal goals of treatment still remain unclear in people with diabetes. ${ }^{45} 10$

The UKPDS study (an early trial which also looked at people with newly diagnosed diabetes) found that tight lowering of blood pressure markedly reduced the incidence of microvascular and macrovascular endpoints in patients with type 2 diabetes. ${ }^{45}$ However, patients assigned to the intensive treatment group $(<150 / 85 \mathrm{~mm} \mathrm{Hg})$ achieved a mean blood pressure of $144 / 82$ $\mathrm{mm} \mathrm{Hg}$, and patients assigned to the conventional treatment group achieved 154/87 mm Hg during follow-up. Another early study, the HOT trial, randomised hypertensive patients to receive intensive control of diastolic blood pressure (to $\leq 80 \mathrm{~mm} \mathrm{Hg}$ ) or conventional control (to $\leq 90 \mathrm{~mm} \mathrm{Hg}$ ). ${ }^{7}$ A retrospective subgroup analysis showed major reductions in cardiovascular outcomes in patients with diabetes in the intensive treatment group, with an achieved blood pressure of 140/81 $\mathrm{mm} \mathrm{Hg}$. Furthermore, the lowest incidence of cardiovascular events occurred at a blood pressure of $138 / 82 \mathrm{~mm} \mathrm{Hg} .{ }^{6}$ Although these early trials shown the benefits of blood pressure lowering in people with diabetes and changed clinical practice, the achieved blood pressure levels in these studies were considerably higher than the target currently recommended by major guidelines. ${ }^{13} 10$

The additional benefits of lowering blood pressure to below 130/80 mm Hg have not been consistently supported by trial evidence. ${ }^{10}$ The ACCORD trial randomised patients with diabetes to receive intensive therapy (to achieve systolic blood pressure $<120 \mathrm{~mm} \mathrm{Hg}$ ) or standard therapy $(<140 \mathrm{~mm} \mathrm{Hg}) .^{13}$ Participants achieved the treatment targets after one year of follow-up, with a mean systolic blood pressure of $119 \mathrm{~mm} \mathrm{Hg}$ in the intensive group and $133 \mathrm{~mm} \mathrm{Hg}$ in the standard group. This trial provided the opportunity for the first time to evaluate the effects of tight control of systolic blood pressure on the incidence of cardiovascular outcomes in people with type 2 diabetes. However, no significant reduction in cardiovascular outcomes was achieved by lowering the systolic blood pressure below $120 \mathrm{~mm} \mathrm{Hg}$, compared with the group in which systolic blood pressure remained above $130 \mathrm{~mm} \mathrm{Hg}$.

On the other hand, intensive therapy seemed to be beneficial for the prevention of non-fatal and total stroke. A recent meta-analysis of prospective controlled trials indicated that the risk of stroke decreased progressively with blood pressure reduction, although this association was not significant for myocardial infarction in people with type 2 diabetes. ${ }^{21}$ Investigators from the Irbesartan Diabetic Nephropathy Trial found that lowering the blood pressure below 120/85 mm Hg in high risk patients with diabetes increased cardiovascular events and all cause mortality. ${ }^{15}$

The results of our observational study extend previous findings, suggesting that lower levels of blood pressure maintained during the first year after diagnosis of diabetes identify a subset of patients with significantly increased risk of death. This association was observed for both systolic and diastolic blood pressure. Our findings are in line with other studies reporting increased risk of poor outcomes associated with tight control of systolic and diastolic blood pressure in high risk patients, albeit at much lower levels than current guidelines. ${ }^{11} 142223$

\section{Strengths and limitations of the study}

Limitations have to be considered in interpretation of our results. In this retrospective analysis, many factors other than blood pressure might have influenced the associations found. Patients were categorised into groups based on their blood pressure levels exclusively, and they may have differed significantly in other risk factors. Although our analyses adjusted for many factors, these adjustments may not have been sufficient and might not have included other unknown factors. A large proportion of patients received lipid lowering and antiplatelet therapy and antihypertensive drugs, including ACEIs, at the time of the diagnosis of diabetes, which might have reduced cardiovascular risk. Furthermore, this could have reduced the potential cardiovascular benefit of antihypertensive treatments, particularly for those patients who had lower blood pressure at baseline.

Because of the observational nature of this study, our findings of increased risk of death related to tight control of systolic and diastolic blood pressure do not imply causality. Furthermore, although we present blood pressure levels corresponding to the lowest risk of mortality, these values are not a recommendation for an optimal treatment goal, and we can only speculate about the underlying mechanisms that explain these associations. Some studies have suggested that tight control of blood pressure might increase cardiovascular risk by the underperfusion of vital organs. ${ }^{10}$ To avoid reducing diastolic blood pressure below a critical level is especially important to ensure coronary flow during diastole. However, some studies have suggested that the increased mortality associated with lower diastolic blood pressure might be associated with some deterioration of general health, because this relation was also evident in patients treated with placebo. ${ }^{24}$

Patients who had low blood pressure at baseline might also have had increased initial cardiovascular risk, resulting in higher mortality rates. To reduce the presence of high risk patients in the low blood pressure categories, we excluded patients from this study who had established heart failure at diagnosis. Similarly, since previous cardiovascular events can both lower blood pressure and increase the risk of further cardiovascular events including death, the associations found could be a confounding effect of established cardiovascular disease. Therefore, we distinguished between patients with and without cardiovascular disease based on their history of myocardial infarction and stroke before diagnosis of diabetes and analysed the associations separately in these groups.

Although concerns about the validity of longitudinal databases in primary care have been raised, the accuracy and completeness of the data recorded in the General Practice Research Database 
has been documented previously and the database is used extensively for health service and epidemiological research. ${ }^{1625} 26$ We were unable to ascertain the cause of death and assess cardiovascular mortality in relation to blood pressure levels. We did not have information on whether patients were taking their antihypertensive drugs. Furthermore, comorbid conditions were inconsistently coded in the database, particularly in the early part of the study period; therefore, we were unable to adjust for underlying comorbid conditions including microvascular complications. However, we adjusted for other indicators of health, including socioeconomic status.

Other strengths of the study included the use of a large sample of unselected patients with newly diagnosed type 2 diabetes and the long follow-up period, with regularly recorded diagnostic, measures, and outcome codes. Prescription data were accurately captured by using the same database software as that used to generate prescriptions by general practitioners. These results, therefore, reflect true associations in the real world setting. Furthermore, our analyses were adjusted for several baseline characteristics that could plausibly be related to treatment or mortality.

\section{Conclusions and policy implications}

In this large observational study, blood pressure below 130/80 $\mathrm{mm} \mathrm{Hg}$ was not associated with reduced risk of all cause mortality in patients with newly diagnosed diabetes, with or without known cardiovascular disease. Furthermore, low blood pressure, particularly below 110/75 $\mathrm{mm} \mathrm{Hg}$, was associated with increased risk for poor outcomes. Although no causality can be implied for these relations, our results suggest that "the lower the better" approach might not apply to blood pressure control beyond a critical level in high risk patients. Since there is currently no robust evidence available for lowering the blood pressure below $130 / 80 \mathrm{~mm} \mathrm{Hg}$ in people with diabetes, it might be advisable to maintain blood pressure between 130-139/80-85 $\mathrm{mm} \mathrm{Hg}$, supported by other therapeutic and lifestyle interventions to improve cardiovascular outcomes in patients with diabetes. ${ }^{10}$

The Department of Primary Care and Public Health at Imperial College thank the National Institute for Health Research (NIHR) Collaboration for Leadership in Applied Health Research and Care (CLAHRC) scheme, NIHR Biomedical Research Centre scheme, and Imperial Centre for Patient Safety and Service Quality for their support. The Department of Health Sciences at Leicester University thank the NIHR CLAHRC scheme for their support. This study uses data from the Full Feature General Practice Research Database, obtained under license from the UK Medicines and Healthcare Products Regulatory Agency (MHRA). The interpretation and conclusions contained in this study are those of the authors alone. Access to the General Practice Research Database was funded through the Medical Research Council's licence agreement with the MHRA.

Contributors: All authors contributed to the design of the study and cowrote the manuscript. EV undertook the analysis and is the guarantor. Funding: This study received funding from the European Community Seventh Framework Programme under grant agreement 277047. Competing interests: All authors have completed the ICMJE uniform disclosure form at www.icmje.org/coi_disclosure.pdf (available on request from the corresponding author) and declare: support from the European Community Seventh Framework Programme for the submitted work; $\mathrm{CM}$ is funded by the Higher Education Funding Council for England and the NIHR; EV and MH are partly funded by the NIHR; no other relationships or activities that could appear to have influenced the submitted work.

Ethics approval: Ethical approval was not required for this study.

Data sharing: No additional data available.

1 Mancia G, De Backer G, Dominiczak A, Cifkova R, Fagard R, Germano G, et al. 2007 Guidelines for the management of arterial hypertension: the task force for the management of arterial hypertension of the European Society of Hypertension (ESH) and of the European Society of Cardiology (ESC). J Hypertens 2007;25:1105-87.

2 Zanchetti A, Grassi G, Mancia G. When should antihypertensive drug treatment be initiated and to what levels should systolic blood pressure be lowered? A critical reappraisal. $J$ Hypertens 2009;27:923-34.

3 Standards of medical care in diabetes-2010. Diabetes Care 2010;33(suppl 1):S11-61. UK Prospective Diabetes Study Group. Tight blood pressure control and risk of macrovascular and microvascular complications in type 2 diabetes: UKPDS 38. BMJ 1998;317:703-13.

5 UK Prospective Diabetes Study Group. Efficacy of atenolol and captopril in reducing risk of macrovascular and microvascular complications in type 2 diabetes: UKPDS 39. BMJ 1998;317:713-20.

6 Zanchetti A, Hansson L, Clement D, Elmfeldt D, Julius S, Rosenthal T, et al. Benefits and risks of more intensive blood pressure lowering in hypertensive patients of the HOT study with different risk profiles: does a J-shaped curve exist in smokers? J Hypertens 2003;21:797-804.

7 Hansson L, Zanchetti A, Carruthers SG, Dahlof B, Elmfeldt D, Julius S, et al. Effects of intensive blood-pressure lowering and low-dose aspirin in patients with hypertension: Principal results of the hypertension optimal treatment (HOT) randomised trial. HOT Study Group. Lancet 1998;351:1755-62.

8 Lewington S, Clarke R, Qizilbash N, Peto R, Collins R. Age-specific relevance of usual blood pressure to vascular mortality: a meta-analysis of individual data for one million adults in 61 prospective studies. Lancet 2002;360:1903-13.

9 Rutter MK, Nesto RW. Blood pressure, lipids and glucose in type 2 diabetes: how low should we go? Re-discovering personalized care. Eur Heart J 2011;32:2247-55.

10 Mancia G, Laurent S, Agabiti-Rosei E, Ambrosioni E, Burnier M, Caulfield MJ, et al. Reappraisal of European guidelines on hypertension management: a European society of hypertension task force document. J Hypertens 2009;27:2121-58.

11 Messerli FH, Mancia G, Conti CR, Hewkin AC, Kupfer S, Champion A, et al. Dogma disputed: can aggressively lowering blood pressure in hypertensive patients with coronary artery disease be dangerous? Ann Intern Med 2006;144:884-93.

12 Estacio RO, Jeffers BW, Gifford N, Schrier RW. Effect of blood pressure control on diabetic microvascular complications in patients with hypertension and type 2 diabetes. Diabetes Care 2000;23(suppl 2):B54-64.

13 Cushman WC, Evans GW, Byington RP, Goff DC Jr, Grimm RH Jr, Cutler JA, et al. Effects of intensive blood-pressure control in type 2 diabetes mellitus. New Engl J Med 2010;362:1575-85.

14 Bakris GL, Gaxiola E, Messerli FH, Mancia G, Erdine S, Cooper-DeHoff R, et al. Clinical outcomes in the diabetes cohort of the international verapamil SR-trandolapril study. Hypertension 2004;44:637-42.

15 Berl T, Hunsicker LG, Lewis JB, Pfeffer MA, Porush JG, Rouleau JL, et al. Impact of achieved blood pressure on cardiovascular outcomes in the irbesartan diabetic nephropathy trial. J Am Soc Nephrol 2005;16:2170-9.

16 Clinical Practice Research Datalink. www.cprd.com/intro.asp.

17 Gray J, Orr D, Majeed A. Use of read codes in diabetes management in a south london primary care group: Implications for establishing disease registers. BMJ 2003;326:1130.

18 Index of Multiple Deprivation. The English Indices of Deprivation 2004 (revised). 2007. www.communities.gov.uk/publications/communities/englishindices.

19 Turner RC, Millns H, Neil HA, Stratton IM, Manley SE, Matthews DR, et al. Risk factors for coronary artery disease in non-insulin dependent diabetes mellitus: United Kingdom Prospective Diabetes Study (UKPDS: 23). BMJ 1998;316:823-8.

20 Hypertension in diabetes study (HDS): II. Increased risk of cardiovascular complications in hypertensive type 2 diabetic patients. J Hypertens 1993;11:319-25.

21 Reboldi G, Gentile G, Angeli F, Ambrosio G, Mancia G, Verdecchia P. Effects of intensive blood pressure reduction on myocardial infarction and stroke in diabetes: A meta-analysis in 73,913 patients. $J$ Hypertens 2011;29:1253-69.

22 Cooper-DeHoff RM, Gong Y, Handberg EM, Bavry AA, Denardo SJ, Bakris GL, et al. Tight blood pressure control and cardiovascular outcomes among hypertensive patients with diabetes and coronary artery disease. JAMA 2010;304:61-8.

23 Bangalore S, Messerli FH, Wun CC, Zuckerman AL, DeMicco D, Kostis JB, et al. J-curve revisited: an analysis of blood pressure and cardiovascular events in the treating to new targets (TNT) trial. Eur Heart J 2010;31:2897-908.

24 Staessen J, Bulpitt C, Clement D, De Leeuw P, Fagard R, Fletcher A, et al. Relation between mortality and treated blood pressure in elderly patients with hypertension: Report of the European working party on high blood pressure in the elderly. BMJ 1989;298:1552-6.

25 Hollowell J. The general practice research database: Quality of morbidity data. Popul Trends 1997:36-40.

26 Chen YC, Wu JC, Haschler I, Majeed A, Chen TJ, Wetter T. Academic impact of a public electronic health database: bibliometric analysis of studies using the general practice research database. PloS One 2011;6:e21404.

Accepted: 12 August 2012

\section{Cite this as: BMJ 2012;345:e5567}

This is an open-access article distributed under the terms of the Creative Commons Attribution Non-commercial License, which permits use, distribution, and reproduction in any medium, provided the original work is properly cited, the use is non commercial and is otherwise in compliance with the license. See: $\mathrm{http} / / /$ creativecommons.org/licenses/bync/2.0/ and http://creativecommons.org/licenses/by-nc/2.0/legalcode. 


\section{What is already known on this topic}

Guidelines recommend that patients at high risk of cardiovascular disease should maintain blood pressure below $130 / 85 \mathrm{~mm} \mathrm{Hg}$ There is no convincing evidence from clinical trials that maintaining a low blood pressure in patients with type 2 diabetes provides additional cardiovascular benefits

Some clinical trials suggest that aggressive lowering of blood pressure may be harmful

\section{What this study adds}

Blood pressure below $130 / 80 \mathrm{~mm} \mathrm{Hg}$ was not associated with reduced risk of all cause mortality in patients with newly diagnosed diabetes, with or without known cardiovascular disease

Low blood pressure, particularly below $110 / 75 \mathrm{~mm} \mathrm{Hg}$, was associated with increased risk for all cause mortality

The "lower the better" approach might not apply to blood pressure control beyond a critical level in high risk patients

\section{Tables}

\begin{tabular}{|c|c|c|c|}
\hline & Patients with cardiovascular disease & Patients without cardiovascular disease & Total \\
\hline No of patients (\%) & $12379(9.8)$ & $113713(90.2)$ & $126092(100)$ \\
\hline No of deaths (\%) & $3535(28.6)$ & $21960(19.3)$ & $25495(20.2)$ \\
\hline Event rate per 1000 patient years & 82.9 & 45.3 & 48.3 \\
\hline Age $\left(\right.$ years) ${ }^{*}$ & $72.9(10.7)$ & $66.4(13.8)$ & $67.1(13.7)$ \\
\hline Male sex & $7178(58.0)$ & $56057(49.3)$ & $63235(50.1)$ \\
\hline Deprivation score & $21.0(22.7)$ & $20.6(22.7)$ & $20.7(22.7)$ \\
\hline \multicolumn{4}{|l|}{ Smoking history } \\
\hline Current smoker & $1866(15.1)$ & $17659(15.5)$ & $19525(15.5)$ \\
\hline Ex-smoker & $4571(36.9)$ & $32393(28.5)$ & $36964(29.3)$ \\
\hline Non-smoker & $5515(44.5)$ & $60205(52.9)$ & $65720(52.1)$ \\
\hline Missing data & $427(3.5)$ & $3456(3.1)$ & $3883(3.1)$ \\
\hline Body mass index ${ }^{*}$ & $28.2(5.4)$ & $29.3(6.1)$ & $29.1(6.1)$ \\
\hline \multicolumn{4}{|l|}{ Blood pressure at diagnosis $(\mathrm{mm} \mathrm{Hg})^{*}$} \\
\hline Systolic & $144.1(21.3)$ & $146.4(21.0)$ & $146.2(21.1)$ \\
\hline Diastolic & $80.3(11.1)$ & $83.2(11.0)$ & $82.9(11.0)$ \\
\hline Diagnosis of hypertension at baseline & $6597(53.3)$ & $48381(42.55)$ & $54978(43.6)$ \\
\hline Systolic blood pressure achieved during first year $(\mathrm{mm} \mathrm{Hg})^{*}$ & $142.4(19.2)$ & $144.3(18.7)$ & $144.1(18.7)$ \\
\hline$<130 \mathrm{~mm} \mathrm{Hg}$ achieved (tight control) & $2773(22.4)$ & $21293(18.7)$ & $24066(19.1)$ \\
\hline 130-139 mm Hg achieved (usual control) & $2515(20.3)$ & $22562(19.8)$ & 25077 (19.9) \\
\hline$\geq 140 \mathrm{~mm} \mathrm{Hg}$ achieved (uncontrolled) & $7091(57.3)$ & $69858(61.4)$ & $76949(61.0)$ \\
\hline Diastolic blood pressure achieved during first year $(\mathrm{mm} \mathrm{Hg})^{*}$ & $79.0(9.6)$ & $81.8(9.5)$ & $81.5(9.5)$ \\
\hline$<80 \mathrm{~mm} \mathrm{Hg}$ achieved (tight control) & $5841(47.2)$ & $39218(34.5)$ & $45059(35.7)$ \\
\hline 80-85 mm Hg achieved (usual control) & $3166(25.6)$ & $31714(27.9)$ & $34880(27.7)$ \\
\hline$\geq 85 \mathrm{~mm} \mathrm{Hg}$ achieved (uncontrolled) & $3372(27.2)$ & $42781(37.6)$ & $46153(36.6)$ \\
\hline $\mathrm{HbA}_{1 \mathrm{c}}$ at baseline $(\%)^{*}$ & $7.2(1.4)$ & $7.4(1.6)$ & $7.4(1.6)$ \\
\hline Total cholesterol at baseline $(\mathrm{mmol} / \mathrm{L})^{*}$ & $4.8(1.1)$ & $5.2(1.3)$ & $5.2(1.4)$ \\
\hline \multicolumn{4}{|l|}{ Anti-hypertensive drug use (at baseline) } \\
\hline Any & $9562(77.2)$ & $57741(50.8)$ & $67303(53.4)$ \\
\hline Monotherapy & $3796(30.7)$ & $29440(25.9)$ & $33236(26.4)$ \\
\hline Multiple therapy & $5766(46.6)$ & $28301(24.9)$ & $34067(27.0)$ \\
\hline \multicolumn{4}{|l|}{ Other drug use (at any time) } \\
\hline Lipid lowering & $5862(47.3)$ & $46638(41.0)$ & $52500(41.6)$ \\
\hline Oral antidiabetic & $5467(44.2)$ & $58893(51.8)$ & $64360(51.0)$ \\
\hline Insulin or oral antidiabetic & $5726(46.3)$ & 61264 (53.9) & $66990(53.1)$ \\
\hline Aspirin or other antiplatelet & $9850(79.6)$ & $52366(46.0)$ & $62216(49.3)$ \\
\hline
\end{tabular}




\section{Table 1 (continued)}

${ }^{*}$ Mean (standard deviation).

†Median (interquartile range). 
Table 2| Risk of all cause mortality in patients newly diagnosed with type 2 diabetes, by level of systolic and diastolic blood pressure

$\begin{gathered}\text { Patients with cardiovascular } \\ \text { disease }\end{gathered}$
Hazard ratio $(95 \% \mathrm{Cl}) \quad$ P

Patients without cardiovascular disease

Hazard ratio $(95 \% \mathrm{Cl})$
Total

Cl)

Systolic blood pressure control $(\mathrm{mm} \mathrm{Hg})$

Model 1 (unadjusted)*

\begin{tabular}{|c|c|c|c|c|c|c|c|}
\hline Tight control 3 & $<110$ & 2.06 (1.67 to 2.55 ) & $<0.001$ & 2.17 (1.94 to 2.42$)$ & $<0.001$ & 2.23 (2.02 to 2.45 ) & $<0.001$ \\
\hline Tight control 2 & $110-119$ & $1.57(1.34$ to 1.84$)$ & $<0.001$ & 1.36 (1.26 to 1.47$)$ & $<0.001$ & $1.41(1.31$ to 1.51$)$ & $<0.001$ \\
\hline Tight control 1 & $120-129$ & $1.13(0.99$ to 1.28$)$ & 0.07 & $1.12(1.05$ to 1.19$)$ & $<0.001$ & $1.12(1.06$ to 1.19$)$ & $<0.001$ \\
\hline Usual control & $130-139$ & 1.00 & 一 & 1.00 & 一 & 1.00 & 一 \\
\hline Uncontrolled 1 & $140-149$ & $1.03(0.92$ to 1.14$)$ & 0.61 & $1.06(1.01$ to 1.11$)$ & 0.02 & 1.05 (1.01 to 1.09$)$ & 0.01 \\
\hline Uncontrolled 2 & $150-159$ & 1.08 (0.96 to 1.22$)$ & 0.21 & $1.14(1.08$ to 1.20$)$ & $<0.001$ & $1.13(1.07$ to 1.18$)$ & $<0.001$ \\
\hline Uncontrolled 3 & $\geq 160$ & 1.22 (1.09 to 1.36$)$ & $<0.001$ & 1.45 (1.38 to 1.53$)$ & $<0.001$ & $1.42(1.35$ to 1.48$)$ & $<0.001$ \\
\hline \multicolumn{8}{|c|}{ Model 2 (adjusted)† } \\
\hline Tight control 3 & $<110$ & 2.79 (1.74 to 4.48$)$ & $<0.001$ & 2.42 (1.66 to 3.52 ) & $<0.001$ & 2.56 (1.89 to 3.47$)$ & $<0.001$ \\
\hline Tight control 2 & $110-119$ & 1.08 (0.71 to 1.62$)$ & 0.92 & $1.58(1.27$ to 1.96$)$ & $<0.001$ & $1.47(1.22$ to 1.76$)$ & $<0.001$ \\
\hline Tight control 1 & $120-129$ & $1.14(0.85$ to 1.54$)$ & 0.38 & $1.06(0.90$ to 1.26$)$ & 0.47 & $1.08(0.93$ to 1.25$)$ & 0.33 \\
\hline Usual control & $130-139$ & 1.00 & 一 & 1.00 & - & 1.00 & - \\
\hline Uncontrolled 1 & $140-149$ & $0.78(0.61$ to 1.01$)$ & 0.06 & $0.91(0.80$ to 1.04$)$ & 0.21 & $0.89(0.79$ to 1.00$)$ & 0.05 \\
\hline Uncontrolled 2 & $150-159$ & $0.81(0.58$ to 1.11$)$ & 0.17 & $1.07(0.91$ to 1.25$)$ & 0.42 & $1.01(0.88$ to 1.15$)$ & 0.89 \\
\hline Uncontrolled 3 & $\geq 160$ & 0.79 (0.57 to 1.09$)$ & 0.15 & $1.16(0.99$ to 1.36$)$ & 0.06 & 1.09 (0.95 to 1.25$)$ & 0.23 \\
\hline \multicolumn{8}{|c|}{ Diastolic blood pressure control (mm Hg) } \\
\hline \multicolumn{8}{|c|}{ Model 1 (unadjusted) ${ }^{*}$} \\
\hline Tight control 3 & $<70$ & 1.54 (1.39 to 1.69$)$ & $<0.001$ & 1.80 (1.69 to 1.91$)$ & $<0.001$ & 1.81 (1.71 to 1.91$)$ & $<0.001$ \\
\hline Tight control 2 & $70-74$ & $1.18(1.06$ to 1.32$)$ & 0.003 & $1.23(1.18$ to 1.29$)$ & $<0.001$ & $1.24(1.19$ to 1.30$)$ & $<0.001$ \\
\hline Tight control 1 & $75-79$ & 0.77 (0.68 to 0.86$)$ & $<0.001$ & 0.87 (0.82 to 0.92$)$ & $<0.001$ & $0.87(0.83$ to 0.91$)$ & $<0.001$ \\
\hline Usual control & $80-84$ & 1.00 & - & 1.00 & - & 1.00 & - \\
\hline Uncontrolled 1 & $85-89$ & 0.87 (0.78 to 0.97$)$ & 0.02 & 0.73 (0.69 to 0.77$)$ & $<0.001$ & 0.75 (0.72 to 0.78$)$ & $<0.001$ \\
\hline Uncontrolled 2 & $90-94$ & 0.99 (0.88 to 1.12$)$ & 0.93 & $0.92(0.88$ to 0.96$)$ & $<0.001$ & $0.92(0.88$ to 0.96$)$ & $<0.001$ \\
\hline Uncontrolled 3 & $\geq 95$ & $1.04(0.91$ to 1.19$)$ & 0.58 & $0.96(0.90$ to 1.02$)$ & 0.81 & $0.95(0.90$ to 1.01$)$ & 0.11 \\
\hline \multicolumn{8}{|c|}{ Model 2 (adjusted) $\dagger$} \\
\hline Tight control 3 & $<70$ & 1.89 (1.40 to 2.56$)$ & $<0.001$ & 1.54 (1.33 to 1.77$)$ & $<0.001$ & 1.59 (1.41 to 1.80$)$ & $<0.001$ \\
\hline Tight control 2 & $70-74$ & 1.32 (1.02 to 1.78$)$ & 0.04 & $1.17(1.02$ to 1.34$)$ & 0.02 & 1.21 (1.07 to 1.37$)$ & 0.002 \\
\hline Tight control 1 & $75-79$ & 0.98 (0.73 to 1.34$)$ & 0.92 & 0.87 (0.76 to 0.99$)$ & 0.04 & 0.89 (0.79 to 1.00$)$ & 0.06 \\
\hline Usual control & $80-84$ & 1.00 & - & 1.00 & - & 1.00 & - \\
\hline Uncontrolled 1 & $85-89$ & $1.31(0.96$ to 1.79$)$ & 0.09 & $0.93(0.81$ to 1.07$)$ & 0.49 & $1.01(0.88$ to 1.14$)$ & 0.92 \\
\hline Uncontrolled 2 & $90-94$ & $1.22(0.82$ to 1.83$)$ & 0.31 & $0.90(0.76$ to 1.07$)$ & 0.32 & $0.96(0.82$ to 1.13$)$ & 0.65 \\
\hline Uncontrolled 3 & $\geq 95$ & $0.93(0.53$ to 1.64$)$ & 0.81 & 1.15 (0.95 to 1.39$)$ & 0.09 & $1.18(0.98$ to 1.43$)$ & 0.07 \\
\hline
\end{tabular}

*Odds ratios were from logistic regression models; proportional hazards assumption was violated.

†Adjusted for age at diagnosis, sex, practice level clustering, socioeconomic deprivation score, body mass index, smoking, baseline $\mathrm{HbA}_{10}$, baseline cholesterol levels, and difference between achieved and baseline blood pressure. 


\section{Figures}
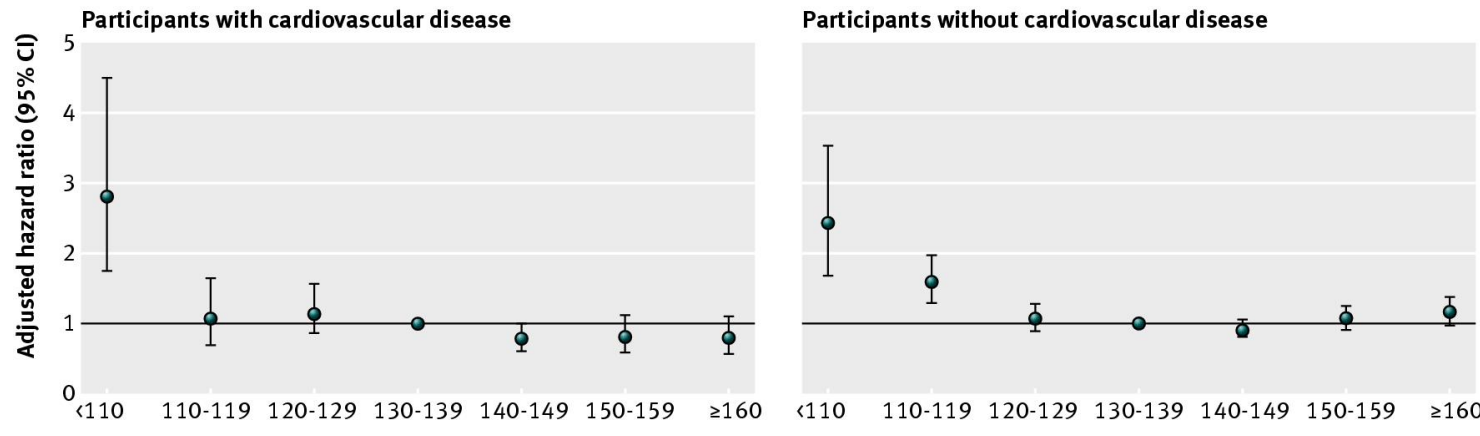

Systolic blood pressure (mm Hg)

Systolic blood pressure $(\mathrm{mm} \mathrm{Hg})$
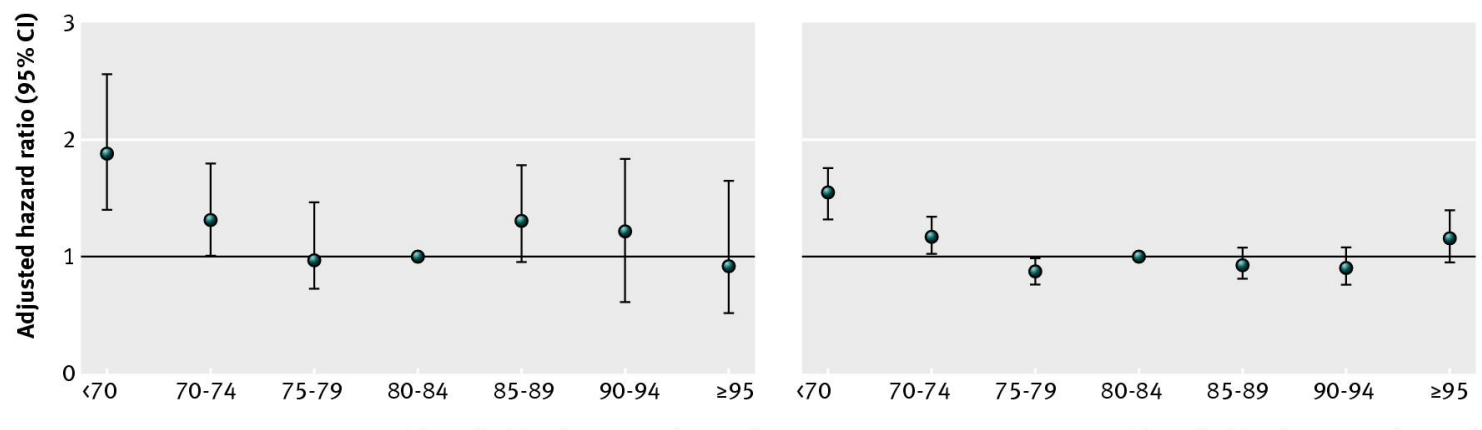

Diastolic blood pressure ( $\mathrm{mm} \mathrm{Hg}$ )

Diastolic blood pressure ( $\mathrm{mm} \mathrm{Hg}$ )

Fig 1 Adjusted risk of all cause mortality in study participants, according to blood pressure level. Cox proportional hazard regression models adjusted for age at diagnosis, sex, practice level clustering, deprivation score, body mass index, smoking, baseline levels of $\mathrm{HbA}_{1 \mathrm{c}}$ and cholesterol, and blood pressure at baseline. 
Participants with cardiovascular disease

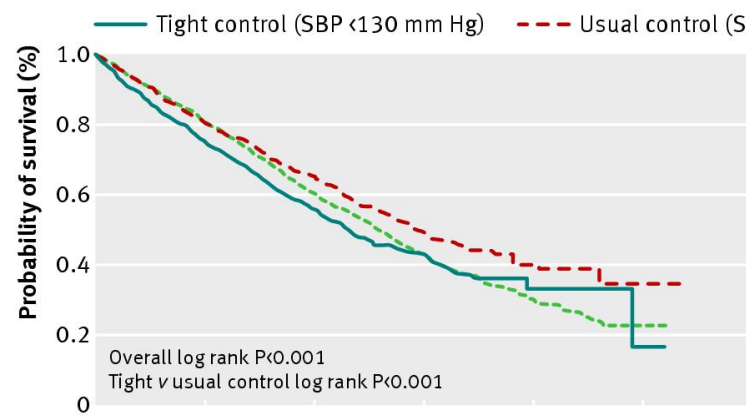

Participants without cardiovascular disease

$30-139 \mathrm{~mm} \mathrm{Hg}$ ) ...-- Uncontrolled (SBP $\geq 140 \mathrm{~mm} \mathrm{Hg}$ )

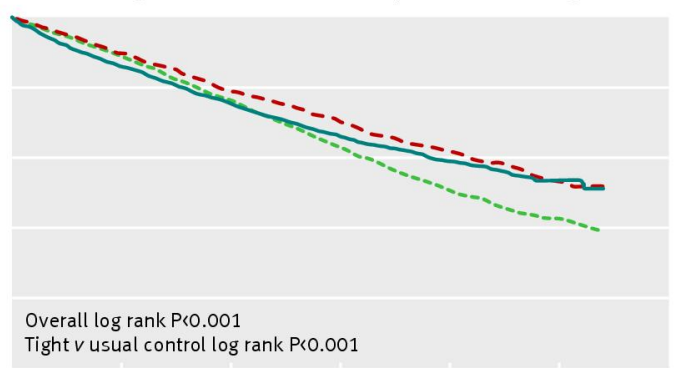

\section{No of patients at risk}

Tight control

\begin{tabular}{|c|c|c|c|c|c|c|c|c|c|c|c|}
\hline $\begin{array}{c}2773 \\
\text { Jsual control }\end{array}$ & 1082 & 288 & 68 & 10 & 1 & $\begin{array}{c}21292 \\
\text { Usual control }\end{array}$ & 10249 & 4197 & 1745 & 597 & 109 \\
\hline $\begin{array}{c}2515 \\
\text { Uncontrolled }\end{array}$ & 1048 & 307 & 89 & 27 & 2 & $\begin{array}{c}22562 \\
\text { Uncontrolled }\end{array}$ & 11502 & 4603 & 1916 & 622 & 85 \\
\hline 7091 & 3826 & 1392 & 426 & 69 & 11 & 69858 & 43091 & 20275 & 8247 & 2321 & 375 \\
\hline
\end{tabular}

Tight control

- - Tight control (DBP $<80 \mathrm{~mm} \mathrm{Hg}$ ) - - Usual control (DBP 80-84 mm Hg) —— Uncontrolled (DBP $\geq 85 \mathrm{~mm} \mathrm{Hg})$
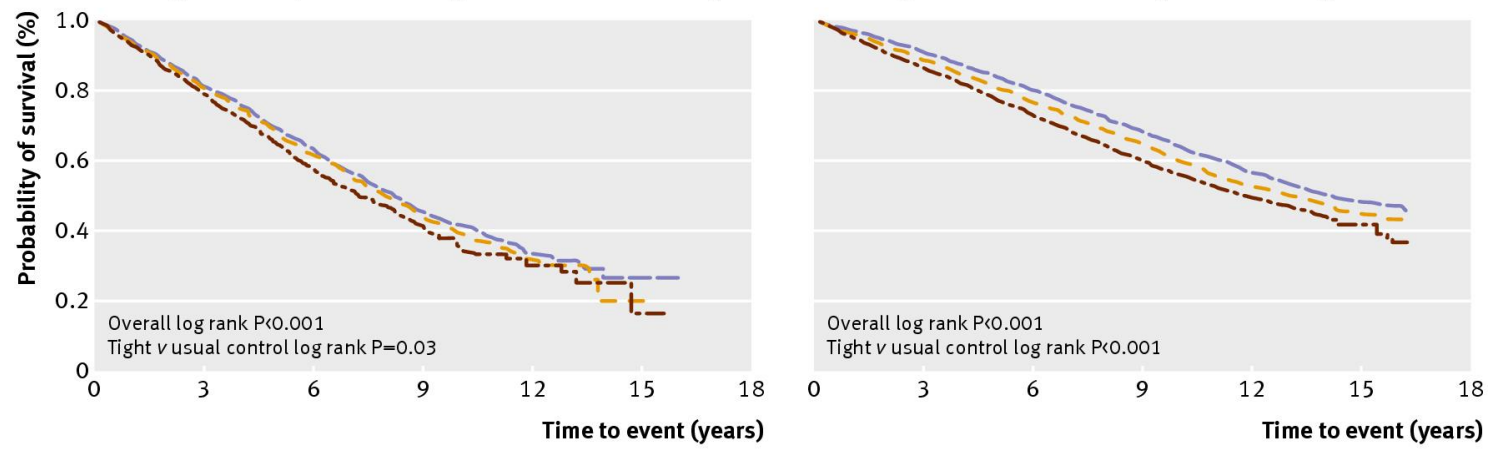

No of patients at risk

No of patients at risk

Tight control

$2773 \quad 1082 \quad 288-68$ Tight contro

$\begin{array}{ccccccc}2773 & 1082 & 288 & 68 & 10 & 1 & \begin{array}{c}21292 \\ \text { Usual control }\end{array} \\ \text { Usual control } & & & & & & \end{array}$

$\begin{array}{lllll}2515 & 1048 & 307 & 89 & 27\end{array}$

Uncontrolled
7091 $3826 \quad 1392 \quad 426 \quad 69 \quad 11$

$\begin{array}{lllll}22562 & 11502 & 4603 & 1916 & 622\end{array}$

Uncontrolled

$69858 \quad 43091 \quad 20275 \quad 8247 \quad 2321 \quad 375$

Fig 2 Kaplan-Meier survival estimates for all cause mortality in study participants with and without cardiovascular disease, according to levels of systolic (SBP) and diastolic (DBP) blood pressure
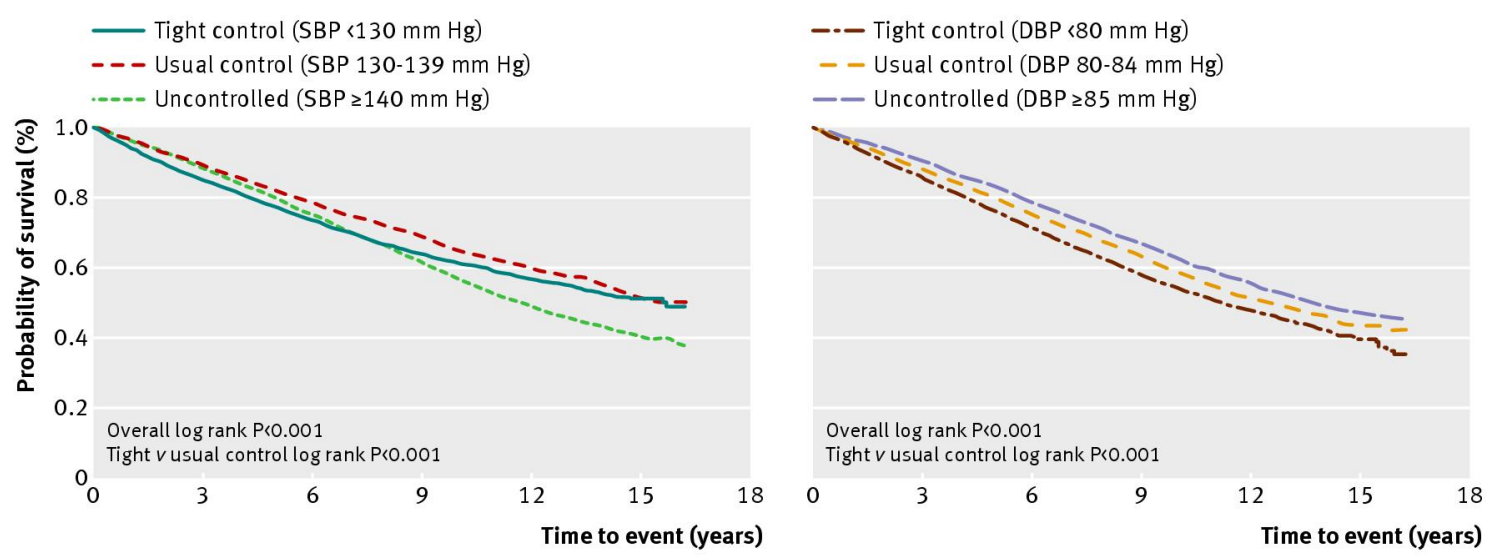

\section{No of patients at risk}

Tight control

$\begin{array}{llllll}24066 & 11331 & 4485 & 1813 & 607 & 110\end{array}$

Usual control

$\begin{array}{llllll}25077 & 12550 & 4910 & 2005 & 649 & 87\end{array}$

Uncontrolled

$\begin{array}{llllll}76949 & 46917 & 21667 & 8673 & 2390 & 386\end{array}$

\section{No of patients at risk}

\begin{tabular}{|c|c|c|c|c|c|}
\hline Tight control & & & & & \\
\hline $\begin{array}{c}45059 \\
\text { Usual control }\end{array}$ & 22426 & 8236 & 3009 & 851 & 118 \\
\hline $\begin{array}{c}34880 \\
\text { Uncontrolled }\end{array}$ & 19990 & 8735 & 3542 & 951 & 125 \\
\hline 46153 & 28382 & 14091 & 5940 & 1844 & 340 \\
\hline
\end{tabular}

Fig 3 Kaplan-Meier survival estimates for all cause mortality according to blood pressure levels in study participants 